\title{
Acción estatal en la producción de servicios sanitarios en localidades de Santa Fe provistas por
} cooperativas

\section{State action in the production of sanitary services in Santa Fe locations provided by cooperatives}

\author{
Orta, Melisa; Forte, Gustavo; Portapila, Margarita
}

Melisa Orta melisaorta@gmail.com

Universidad Nacional de Rosario, Argentina

Gustavo Forte gusforte@retina.ar

Universidad de Buenos Aires, Argentina

Margarita Portapila margarita.portapila@gmail.com Consejo Nacional de Investigaciones Científicas y Técnicas - Universidad Nacional de Rosario, Argentina

Estudios Sociales. Revista Universitaria Semestral Universidad Nacional del Litoral, Argentina

ISSN: 0327-4934

ISSN-e: 2250-6950

Periodicidad: Semestral

núm. 61, e0005, 2021

estudiossociales@unl.edu.ar

Recepción: 06 Febrero 2020

Aprobación: 30 Julio 2021

URL: http://portal.amelica.org/ameli/jatsRepo/293/2932743008/ index.html

DOI: https://doi.org/10.14409/es.2021.2.e0005

El contenido está bajo Licencia Creative Commons AtribuciónNoComercial-Compartir Igual 4.0 Internacional. Atribución - No Comercial - Compartir Igual (BY-NC-SA): no se permite un uso comercial de la obra original ni de las posibles obras derivadas, la distribución de las cuales se debe hacer con una licencia igual a la que regula la obra original.

\section{(i) (2)}

Esta obra está bajo una Licencia Creative Commons AtribuciónCompartirIgual 4.0 Internacional.
Resumen: En el presente trabajo, analizamos cómo participa el Estado de la provincia de Santa Fe (Argentina), en el proceso de producción social de servicios de agua y saneamiento en aquellas localidades que son provistas por cooperativas. Nos interesa observar si se produce un cambio en el modo en cómo se relacionan los actores del orden gubernamental-institucional y las entidades cooperativas a partir de que el Partido Socialista asume el poder ejecutivo provincial. Identificamos cuáles fueron los actores involucrados, los conflictos que se producen y las estrategias que desarrollan para universalizar el acceso a estos servicios públicos esenciales.

Palabras clave: acción estatal, servicios de agua y saneamiento, Santa Fe, cooperativas.

Abstract: In this paper, we analyse how the State of the Santa Fe province (Argentina) participates in the process of social production of water and sanitation services, in those localities supplied by cooperatives. We are interested in observing whether there is a change in the relationship between the governmental-institutional order and the cooperative entities, from the moment in which the Socialist Party takes over the provincial administration. We identify the involved actors, the conflicts that occur and the strategies they develop to universalize access to these essential public services.

Keywords: state action, water and sanitation services, Santa Fe, cooperatives.

\section{Introducción}

A nivel internacional, en las últimas décadas tuvo lugar un incremento progresivo la importancia que ha adquirido el problema de garantizar la universalización del 
acceso a los Servicios de Agua y Saneamiento (SAS). En este marco, Naciones Unidas (NU) estableció en 2010 que el acceso seguro a agua potable salubre y al saneamiento constituye un derecho humano fundamental para el completo disfrute de la vida y de todos los demás derechos humanos (Resolución 64/292 de 2010). Sin embargo, a pesar de que se han fijado metas y objetivos que propendieron a universalizar el acceso a estos servicios básicos, aún persisten desigualdades socio-económicas, geográficas, culturales y entre regiones urbanas y rurales. De manera creciente, la literatura considera que los desafíos para garantizar el Derecho Humano al Agua y Saneamiento (DHAS) no son sólo de carácter natural, técnico o ni siquiera económico, sino que tienen que ver también con el modo en cómo se gobiernan y gestionan estos servicios y con las relaciones sociales de poder que se constituyen y que posibilitan o dificultan la efectividad de las políticas públicas que tienden a la concreción de estos derechos (Castro, 2007). En este sentido, el análisis del ámbito de las relaciones sociales estatales en el proceso de producción social de agua y saneamiento adquiere una importancia fundamental para poder identificar cuáles son las condiciones necesarias para hacer efectivo el DHAS.

La elección del caso de estudio se debe a que en la actualidad la provincia de Santa Fe (Argentina) se caracteriza por poseer un sistema discriminatorio de gestión de los SAS, en el cual el Estado provincial solamente se hace cargo de proveer el servicio de forma directa a las 15 localidades más pobladas, con tarifas subsidiadas e inversión pública en infraestructura. De las 347 poblaciones restantes, en 2007 nos encontramos con que 149 eran provistas por sus estados locales, 76 por cooperativas, 46 tenían una prestación mixta (cooperativa y Estado local) y 76 localidades pequeñas no contaban con prestación centralizada del servicio. El marco normativo no establece la obligación de subsidiar a estas 347 poblaciones que están por fuera del territorio a cargo del Estado provincial.

En este trabajo, nos interesa analizar la situación de las localidades de la provincia de Santa Fe, cuyos principales proveedores de SAS son cooperativas ¿Por qué esta decisión? Los gobiernos locales tienen mayor posibilidad de acceder a recursos económicos debido a su potestad tributaria y a la recepción de fondos provenientes de la coparticipación provincial, siendo las cooperativas los proveedores más postergados en cuanto a la atención estatal. Por otro lado, debido a que el poder de concesión está a cargo de los Estados locales, las cooperativas, a diferencia de las comunas y municipios, están sujetas a un doble control: por parte de las autoridades locales y por parte del Ente Regulador de los Servicios Sanitarios (ENRESS). Esto dificulta los trámites necesarios que debe hacer una cooperativa para poder funcionar, como por ejemplo el pedido de aumento de tarifa.

Para analizar este proceso, partimos de una concepción del Estado como una relación social compleja. Esta concepción, nos conduce a realizar un análisis que involucre el accionar del Estado inscripto en la totalidad del proceso social que estamos estudiando, permitiendo no sustraer al mismo su indudable especificidad y relevancia en la configuración de las relaciones sociales. Hablar de relaciones sociales supone identificar cuáles son las distintas acciones sociales que se ponen en correspondencia, quiénes son los actores que las llevan adelante, los conflictos que se establecen y las relaciones de poder entre estos actores. Se prestará atención 
principalmente a las estrategias que desarrolla la rama del Poder Ejecutivo Provincial (PEP) para garantizar el acceso a bienes colectivos básicos.

En el primer apartado de este trabajo describimos brevemente aspectos estructurales de la situación actual del modelo de gestión de SAS en la provincia y el marco normativo e institucional que regula el desempeño de las cooperativas. En el segundo apartado, especificamos el marco teórico del que partimos y las preguntas de investigación que se derivan de este enfoque particular. En el tercer apartado detallamos y justificamos la propuesta metodológica adoptada, basada en la reconstrucción de procesos a partir del relevamiento de la prensa escrita. El cuarto apartado constituye un estudio empírico exploratorio, compuesto a partir del análisis de una base de datos conformada por el relevamiento de noticias, cuyas variables intentan responder a los interrogantes teórico-empíricos que nos planteamos. Complementamos esta información con la realización de entrevistas a actores clave y el análisis de documentos institucionales. Por último, presentamos algunas conclusiones preliminares a partir de los resultados obtenidos mediante el análisis de la información relevada.

\section{Sistema de provisión de los Servicios de Agua y Saneamiento (SAS) en Santa Fe}

La provincia de Santa Fe (Argentina) tiene una superficie de más de 130.000 $\mathrm{km}^{2}$ y una población de más de 3 millones de habitantes. Está dividida política y administrativamente en 19 departamentos que a su vez se subdividen en 362 distritos. De estos distritos, 50 son municipios (más de 10.000 habitantes) y 312 asumen la forma de comunas (menos de 10.000 habitantes). Está ubicada en la región centro-este de la Argentina e integra una de las regiones agropecuarias e industriales más importantes del país. Cuenta con una participación del Producto Bruto Geográfico (PBG) del $8 \%$ en el total nacional, siendo la segunda provincia en importancia después de Buenos Aires (IPEC, 2013). Sin embargo, a pesar de su importancia económica, presenta históricamente un desarrollo asimétrico y desigualdades socio-estructurales profundas (Urcola, Nogueira, Porstmann y Lattuada, 2015).

Una de las desigualdades que está presente en la provincia tiene que ver con el sistema actual de gestión de los SAS en Santa Fe, que se fue configurando a lo largo de un proceso histórico ${ }^{[1]}$. Está regulado por la Ley $11220 / 94$, que determina un esquema de gestión discriminatorio de los servicios sanitarios, puesto que el Estado provincial está obligado a prestar los servicios de agua y cloacas solamente a 15 localidades, que juntas reúnen el 59\% de la población provincial. En las 347 localidades restantes el poder de concesión está a cargo de sus gobiernos locales y el gobierno provincial no está obligado a garantizar con recursos propios que el poblamiento de estas localidades acceda a los SAS (Orta, Portapila, Antón y Forte, 2020).

La empresa Aguas Santafesinas SA (ASSA), de capital accionario de mayoría del Estado provincial y encargada de proveer los SAS en esas 15 localidades, cuenta con subsidios que alcanzan a cubrir el $40 \%$ de sus gastos operativos mientras que los operadores de las 347 localidades restantes no tienen ningún tipo de subsidio por parte del Estado provincial. Esto genera fuertes inequidades en términos de los recursos (económicos, institucionales, tecnológicos y de 
conocimiento) con las que cuentan los pequeños y medianos prestadores para hacer frente a los problemas de producción y provisión de agua para consumo humano en los territorios que están a su cargo. Entre estos pequeños y medianos prestadores están presentes en la provincia 126 cooperativas de SAS que nacieron mediante la iniciativa de los vecinos de las localidades rurales, que no contaban con los servicios básicos. De esta manera, en el año 2007, cuando comienza nuestro período de estudio, existía un sistema de gestión de los SAS en el cual convivían tres subsistemas: 15 localidades provistas por ASSA; 271 localidades provistas por otros prestadores y 76 localidades sin prestación centralizada del servicio (ver Cuadro 1).

\section{Cuadro 1.}

Sistema de gestión de los SAS en Santa Fe. Ley Provincial 11220/94

\begin{tabular}{|lccc|}
\hline \multicolumn{1}{c}{ Subsistema } & Localidades & Población & $\%$ población \\
\hline ASSA & 15 & 1.888 .237 & 59 \\
Otros prestadores & & & \\
(126 cooperativas) & 271 & 1.287 .229 & 38 \\
Sin red & 76 & 19.071 & 3 \\
\hline Total & 362 & 3.194 .537 & 100 \\
\hline
\end{tabular}

Fuente: elaboración propia en base al CNPHV 2010 y ENRESS (2016).

En cuanto al acceso a los servicios de agua y saneamiento, también se presentan desigualdades según quién sea el prestador de SAS. Las poblaciones donde el prestador principal es una cooperativa, tienen un promedio de acceso a agua por red pública del $80 \%$ y del $22 \%$ en cloacas. Esta situación contrasta con la realidad de las localidades que son provistas por la empresa estatal, donde el promedio de acceso a agua por red pública es del $90 \%$ y el de acceso a cloacas es del $50 \%$.

Como mencionamos anteriormente, existen en la provincia 126 cooperativas que proveen los servicios de agua y/o saneamiento en 124 localidades de la provincia. En el cuadro 2 se puede observar que las cooperativas proveen el servicio de agua en el $79 \%$ de las localidades (98 casos) y proveen los servicios de agua y cloacas en un $21 \%$ de las localidades (26 casos). Ahora bien ¿cómo colaboran los gobiernos locales en las localidades donde el prestador principal es una cooperativa? Los municipios y comunas intervienen en la prestación de SAS en 48 de las 122 localidades donde el prestador principal es una cooperativa.

En el Cuadro 3 podemos observar que de este total de 48 localidades, en un $21 \%$ (10 casos) prestan el servicio de agua, complementando el servicio provisto por la cooperativa y en un $79 \%$ (38 casos) prestan el servicio de cloacas.

\section{Cuadro 2.}

Servicios que prestan las cooperativas de SAS

\begin{tabular}{lccc|}
\hline Tipo de prestador & Agua & Agua y cloacas & Total \\
\hline Cooperativa & $79 \%$ & $21 \%$ & $100 \%$ \\
\hline
\end{tabular}

Fuente: elaboración propia en base a ENRESS (2016) 


\section{Cuadro 3.}

Servicios que prestan los gobiernos locales en localidades con cooperativas de SAS

\begin{tabular}{|lccc|}
\hline Tipo de prestador & Agua & Cloacas & Total \\
\hline Gobierno local & $21 \%$ & $79 \%$ & $100 \%$ \\
\hline
\end{tabular}

Fuente: elaboración propia en base a ENRESS (2016)

Del análisis de los cuadros 2 y 3 podemos ver que las cooperativas suministran mayoritariamente agua y un porcentaje mucho menor de servicios de cloacas. La participación de los poderes ejecutivos locales viene a cubrir esta deficiencia ¿Por qué en un $79 \%$ de las localidades provistas por cooperativas, éstas no han podido incorporar en su prestación el servicio de saneamiento? ¿Tiene que ver con aspectos técnicos, económicos o de conocimiento? Estas preguntas fueron abordadas a partir de la realización de entrevistas a actores clave. En una de las entrevistas, realizada a la Directora de la Dirección General de Programas Estratégicos de la Secretaría de Aguas, la funcionaria nos respondió lo siguiente:

«Las localidades no tienen cloacas a falta de proyectos, a falta de políticas públicas en las comunas. Para poder ejecutar una obra necesitas primero tener un proyecto para que luego puedas salir a buscar fondos. Son obras que en general los costos no los pueden afrontar las localidades solas y sobre todo las que son pequeñas. En general lo que falta es falta de proyectos que encaren las comunas. Las cooperativas y prestadores no tienen fondos para afrontar estas obras tan grandes. En general las localidades chicas no tienen equipos de planificación ni equipos de proyectos. El factor económico también es importante. Tampoco hay una planificación general» ${ }^{[2]}$.

A partir de lo mencionado por la entrevistada, podemos observar que las dificultades principales que obstaculizan la provisión del servicio de cloacas por parte de las cooperativas en las localidades pequeñas y medianas se relaciona con la falta de recursos económicos para poder realizar estas obras con fondos propios y la inexistencia de equipos técnicos que les permitan elaborar proyectos para conseguir financiamiento. Otro aspecto que señala está relacionado con la falta de una planificación a nivel provincial.

La política hídrica impulsada por el nuevo gobierno fue definida en el Plan Estratégico Provincial elaborado en 2008 y actualizado en 2012. En este Plan, se definen tres estrategias dirigidas a garantizar el derecho humano a consumir agua de calidad. Estas son: (1) el Sistema Provincial de Acueductos; (2) obras de mejoramiento de la calidad del agua y; (3) obras de provisión de agua potable para las localidades que no cuentan con prestación centralizada del servicio (Gobierno de la Provincia de Santa Fe, 2012: 224). Sin embargo, en el plan estratégico no se menciona ningún objetivo relacionado con la construcción y extensión de redes cloacales. En el análisis de la estrategia selectiva del gobierno provincial, pudimos constatar esta situación, ya que identificamos acciones del PEP para mejorar el acceso al agua potable pero no identificamos acciones tendientes a afrontar la problemática del saneamiento.

Como mencionamos, en diciembre del 2007 se produce un cambio de la fuerza política que ocupa el poder ejecutivo del Estado. Después de más de 24 años de gestión del Partido Justicialista (PJ) asume la gobernación de Santa Fe la alianza denominada Frente Progresista Cívico y Social (FPCyS), que presenta como candidato a gobernador a un miembro del Partido Socialista (PS). La 
nueva gestión dio inicio a un proceso de cuestionamiento y redefinición de los lineamientos principales de la política pública en torno a los servicios de agua y saneamiento en la provincia y diseñó de una nueva política hídrica para Santa Fe, en la cual uno de sus objetivos principales era el de garantizar el derecho humano al agua en todo el territorio provincial (Gobierno de la Provincia de Santa Fe, 2012: 224).

Asimismo, el gobierno provincial se propuso modificar la Ley 11220/94 para crear un marco normativo que reconozca el DHAS y configurar un nuevo sistema de gestión del agua a partir del cual el Estado provincial asumiría la responsabilidad de captar agua cruda, tratarla y transportarla por medio de acueductos hasta cada localidad, mientras los municipios, comunas o cooperativas asumirían la responsabilidad de su potabilización final y su distribución al interior de sus territorios (Orsolini, 2013).

En este contexto estructural: ¿cómo haría la nueva fuerza para garantizar y/o ampliar el derecho humano a los SAS que anunciaba? ¿Comenzó a configurarse un modo distinto de relación entre el poder ejecutivo provincial y las entidades cooperativas? A los fines de abordar estas cuestiones, en los apartados siguientes especificaremos aspectos del marco teórico y metodológico del que partimos.

\section{Marco teórico: abordaje de la acción estatal como una relación social compleja}

Para poder abordar el modo en el que se desempeña el Estado existen distintos enfoques teóricos, que podemos agrupar bajo dos categorías: enfoques estadocéntricos y enfoques socio-céntricos. A los fines de nuestra investigación, adoptamos la propuesta teórica de Bob Jessop, que se propone superar esta dicotomía, permitiendo al mismo tiempo no sustraer al Estado su especificidad y relevancia en la configuración de las relaciones sociales. El autor propone analizar la acción estatal desde una perspectiva relacional de la naturaleza del Estado que habilite interpretar las capacidades e intervenciones estatales y sus consecuencias tomando en consideración el complejo campo de relaciones sociales que las configuran como tales. Más que reificar al Estado como un sujeto homogéneo, implica concebirlo como un ámbito donde conviven distintas relaciones sociales de poder. Esto es, partir de una concepción del Estado como relación social que incorpore como fundamento constitutivo las acciones que desarrollan y las condiciones que contribuyen a generar otros actores sociales que se vinculan con él, incidiendo sustantivamente en la naturaleza y características de las intervenciones estatales (Borrastero, 2018: 20-21).

Jessop retoma a los autores institucionalistas que definen al Estado a partir de la presencia de tres elementos: aparato institucional, territorio y población (en particular Weber). Analiza la relación que existe entre estos tres elementos constitutivos y establece la definición siguiente:

«El núcleo del aparato estatal está compuesto por un conjunto relativamente unificado de instituciones y organizaciones empotradas en la sociedad y formalizadas socialmente y que son estratégicamente selectivas [aparato estatal], cuya función socialmente aceptada es la de definir y aplicar decisiones colectivas vinculantes para los miembros de una sociedad [población] de una determinada área territorial [territorio] en nombre del interés común o la voluntad general de una comunidad 
política imaginada que se identifica con ese territorio [idea de Estado]» (Jessop, 2017: 93).

En su definición de Estado, el autor propone considerar un cuarto elemento, la «idea de Estado», entendido como el proyecto estatal que define la naturaleza y las finalidades de su acción y que sirve para justificar la legitimidad del monopolio del uso de la fuerza física. Jessop afirma que la territorialización del poder político y la creación de la población sobre la que se ejerce ese poder son logros históricos fundados en luchas y, por tanto, son contingentes, están sujetos a los cambios en los equilibrios de poder resultantes de esas luchas.

A partir de esta concepción general del Estado, Jessop propone un marco analítico específico para su estudio. Este enfoque profundiza en el análisis de esas luchas, esas confrontaciones sociales que imponen visiones determinadas, que con el tiempo se consolidan y se cristalizan en instituciones. En este sentido, Jessop se apoya en los teóricos marxistas (particularmente Poulantzas) que proponen abandonar la visión de Estado como cosa o sujeto simple y comenzar a analizar el poder del Estado como una relación social compleja, como una condensación material de relaciones de fuerzas (de poder) entre las clases y fracciones de clases (Jessop, 2008a: 123).

Entonces, para poder estudiar el ámbito de las relaciones sociales estatales, Jessop nos propone partir de un Enfoque Estratégico Relacional (EER), que reconoce la interacción compleja y contingente entre actores e instituciones políticas y el resultado indeterminado de los procesos de políticas resultantes de esta interacción. En este sentido, Jessop argumenta que el ejercicio del poder enmascara un complejo conjunto de relaciones sociales que se extienden mucho más allá del sistema estatal y sus capacidades distintivas por lo que los poderes del Estado siempre serán condicionados o relacionales, dependiendo de los vínculos estratégicos entre los administradores del Estado y otras fuerzas políticas y sociales. En este sentido, las estrategias impulsadas por el Estado, entendidas como proyectos políticos y políticas públicas (cursos de acción), no pueden ser coherentes ya que están atravesadas por esta lucha entre las distintas fracciones al interior del Estado y las relaciones que se establecen con los actores no estatales. Esto se materializa con que lo definido en una rama o aparato de Estado es contradicho o complementado por otra y viceversa, sin estar garantizada en ningún caso una coherencia institucional, como lo pretende mostrar el institucionalismo tradicional. A este modo de entender el accionar del Estado, Jessop lo define como selectividad estratégica. Las acciones (o inacciones del Estado) reflejan el mayor acceso que tienen algunas fuerzas sociales al aparato del Estado y la mayor probabilidad de que sus demandas sean satisfechas. Siguiendo a Jessop, un proyecto hegemónico dado privilegia ciertos intereses particulares compatibles con su concepción del interés general y deroga otros intereses particulares en conflicto o contradictorios (Jessop, 2008b: 30).

La adopción del EER de Jessop, nos conduce a realizar un análisis que involucre el accionar del Estado en la totalidad del proceso social que estamos estudiando, lo que significa prestar atención al modo en cómo se relacionan los actores o fuerzas políticas al interior de Estado y con los actores no estatales y/o fuerzas sociales. Hablar de relaciones sociales supone identificar cuáles son las distintas acciones sociales que se ponen en correspondencia, quiénes son los actores que las llevan adelante, los conflictos que se establecen y las relaciones de poder entre 
estos actores. Se prestará atención principalmente a las estrategias que desarrolla la rama del Poder Ejecutivo Provincial para garantizar el acceso a bienes colectivos básicos.

A partir de los lineamientos teóricos mencionados, nos preguntamos: ¿cuáles son los actores que están involucrados en el proceso social de provisión de SAS? ¿Cuáles son las acciones y las estrategias que desarrollan estos actores, en particular la fuerza política que ocupa el ejercicio del Poder Ejecutivo del Estado provincial, para enfrentar el problema del acceso al agua y saneamiento? ¿Cuáles son los conflictos y las luchas que se establecen entre los actores estatales y no estatales? ¿Comenzó a configurarse un modo distinto de relación entre el Poder Ejecutivo Provincial y las entidades cooperativas? ¿Se superaron, a partir de la nueva gestión del FPCyS, las desigualdades en el acceso a los servicios de agua y saneamiento presentes en la provincia?

En el apartado siguiente, especificamos cuál es la metodología adoptada para poder abordar estas preguntas.

\section{Metodología}

Para poder abordar el accionar del Estado provincial inserto en una complejidad de relaciones sociales, se adoptó una metodología de reconstrucción de procesos a partir de la prensa escrita, utilizada en diversas investigaciones de América Latina sobre conflictividad social ${ }^{[3]}$. Siguiendo el objetivo de observar si se modifica la relación del Poder Ejecutivo del Estado provincial con el poblamiento de esas localidades que han sido pospuestas, particularmente las que son provistas por cooperativas, conformamos una base de datos de noticias, integrada por las dimensiones siguientes: localización espacio-temporal de la acción, sujeto que realiza la acción, forma de acción, destinatario de la acción, contra quién se realiza la acción, problema que origina la acción y orden en el que se inscribe el problema (social, económico, político o ambiental). La base de datos conformada de esta manera constituye un modo de acceder al proceso de la producción social de agua y saneamiento, inscripto en una totalidad, posibilitando la identificación de actores involucrados y las interrelaciones que se establecen entre ellos en una dimensión espacio-temporal.

Seleccionamos dos diarios de relevancia de la provincia, La Capital de Rosario y El Litoral de Santa Fe. Las noticias fueron buscadas en internet a partir de palabras claves ${ }^{[4]}$ durante el período 2007-2015. Esta búsqueda nos arrojó como resultado 683 noticias. Para poder tener un primer acercamiento exploratorio del proceso social que estamos estudiando seleccionamos 150 noticias correspondientes a los años 2010 y 2011. Partiendo de nuestro objetivo de conocer en primer lugar los procesos sociales en los cuales participan las cooperativas, seleccionamos únicamente aquellas noticias en las que estuvieran involucradas algunas de estas entidades - ya sea como sujeto de la acción o destinatario- o que tuvieron lugar en localidades que son provistas por este tipo de instituciones. Obtuvimos un total de 48 noticias.

El recorte temporal se debió a que fueron los dos años en los cuales la prensa analizada reflejó mayor cantidad de noticias y a que tuvieron lugar una serie de hechos sociales y políticos significativos sobre el problema que estamos 
estudiando. Una lectura inicial de las noticias nos permitió relevar los siguientes hechos:

a) A fines de diciembre de 2009 fueron designados los miembros para conformar el directorio del ENRESS, el cual estuvo acéfalo por más de dos años.

b) En marzo de 2010, el ENRESS realizó por primera vez, luego de 12 años de estar en funcionamiento, una Audiencia Pública para discutir los temas referidos a la gestión de los SAS en Santa Fe.

c) En agosto de 2010, se inauguró el primer acueducto construido en la provincia, para proveer de agua potable de calidad a 12 localidades del interior.

d) Durante los años 2010 y 2011, tuvo lugar un período de sequía y escasez de agua que afectó principalmente a las localidades del norte santafesino.

e) En noviembre de 2010, el gobierno provincial presentó un proyecto para modificar el marco regulatorio de SAS fijado por la Ley 11220. Uno de los objetivos del proyecto era establecer un régimen normativo que reconozca el DHAS.

Se construye entonces, la matriz de datos cuya unidad de registro es la acción. Entonces, el problema que enfrentamos fue identificar y destacar las acciones en cada noticia. Tuvimos que transformar esa serie de noticias en una cantidad n de acciones. De las 48 noticias seleccionadas para los años 2010 y 2011 se identificaron 115 acciones. Por último, para el análisis de estas 115 acciones conformamos una base de datos en la cual consideramos las dimensiones mencionadas anteriormente.

En el análisis de estos dos años logramos identificar las cuestiones principales para responder a nuestros objetivos: actores involucrados, modos de relación entre los actores, estrategia predominante del gobierno provincial, modos en los que participa la sociedad civil, posibilidad real o potencial de alianzas entre actores.

Para completar el período de estudio y tener una mayor comprensión de los tipos de relaciones predominantes, los conflictos y los problemas que originan las acciones realizamos entrevistas a actores clave (funcionarios políticos, personal técnico y miembros de organizaciones sociales), analizamos documentos institucionales y relevamos los datos de cobertura de agua y cloacas provistos por el Instituto Nacional de Estadísticas y Censos (INDEC) y por el ENRESS. Esta información nos sirvió para poder identificar la continuidad de la estrategia selectiva del gobierno provincial presente en el sub-período 2010-2011 y desarrollar el conflicto que se suscitó a partir de la presentación del proyecto oficialista para modificar el marco regulatorio.

\section{Análisis empírico}

A continuación, presentamos algunos resultados preliminares, respondiendo a los interrogantes fundamentales que atraviesan nuestra investigación.

1. ¿Quiénes fueron los actores que llevaron adelante el proceso de producción social de los SAS en las localidades de Santa Fe provistas por cooperativas? 
Una primera aproximación al análisis de nuestra base de datos nos permitió constatar la complejidad que posee el proceso social de producción de servicios de agua y saneamiento en el subsistema de localidades provistas por cooperativas, debido a que están involucrados una diversidad de actores en las acciones que identificamos.

Con relación a los actores comprendidos dentro del sistema políticoinstitucional pudimos identificar que estuvieron involucrados desde el gobernador de la provincia hasta los presidentes comunales de pequeñas localidades del norte santafesino. Asimismo, nos encontramos con la participación de funcionarios públicos pertenecientes al PEP, tales como el ministro de Aguas, Servicios Públicos y Medio Ambiente (MASPyMA), el presidente del Ente Regulador de los Servicios Sanitarios (ENRESS) e intendentes de localidades medianas. También estuvieron implicados actores pertenecientes al Poder Ejecutivo Nacional, particularmente la Presidencia de la Nación y el Ente Nacional de Obras Hídricas de Saneamiento (ENOHSA). Además, nos encontramos con actores pertenecientes a fuerzas políticas del partido oficialista o de la oposición, que fueron electos por el voto popular para ocupar cargos legislativos: concejales municipales, senadores y diputados provinciales del Frente Progresista Cívico y Social (FPCyS), del Partido Justicialista (PJ) y del Frente para la Victoria (FpV).

En cuanto a la sociedad civil, las acciones tendientes a resolver los distintos problemas relacionados con la producción de los servicios de agua y saneamiento agrupan a actores con diversos grados de organización. Nos encontramos con la Federación Nacional de Cooperativas de Agua Potable (FeNCAP), asociaciones de defensa de los derechos de usuarios y consumidores, colegios profesionales, la Cátedra Libre del Agua de la Universidad Nacional de Rosario (UNR), todas ellas con un mayor grado de organización. Con un nivel menor de organización estuvieron involucrados periodistas, profesores de la Universidad Nacional del Litoral (UNL), productores rurales y vecinos.

Por último, con relación a los prestadores de los SAS, nos encontramos con acciones realizadas por las cooperativas de SAS o por miembros de los poderes ejecutivos locales, que comparten la prestación del servicio con las entidades cooperativas. También encontramos la participación del presidente de Aguas Santafesinas SA (ASSA) en aspectos relacionados con el marco normativo de los SAS en Santa Fe o en la inauguración de obras que benefician a localidades provistas por el sistema cooperativo.

Una vez presentado el mapa general de los actores involucrados, nos interesa observar cuál es el grado de protagonismo de cada uno de ellos. Esta cuestión será abordada en la siguiente sección.

2. ¿Cuál fue el protagonismo de los actores en la totalidad de los hechos registrados?

Para poder dar cuenta de esta pregunta debimos realizar un agrupamiento de los actores que estuvieron involucrados. Decidimos clasificar esos actores en dos grandes campos, Orden Gubernamental Institucional (OGI) y Sociedad Civil (SC). Dentro de la SC, aquellos actores que participan insertos en el marco de una organización fueron caracterizados como Identidades Político-Sociales (IPS). A los actores de la sociedad civil que intervienen fuera del marco de una 
organización, o que se organizan frente a una situación específica y luego se disgregan, los denominamos actores individuales (AI).

Se encontró que los actores con mayor protagonismo dentro del proceso social de producción de SAS, pertenecen al ámbito superestructural, es decir ocupan las distintas ramas del Estado, representando un 64\% del total de las acciones registradas.

Ahora bien, una vez presentada esta tendencia general, nos interesa conocer cuáles son los actores que más se manifiestan al interior del OGI. En el Cuadro 4 se puede observar que el agrupamiento con mayor grado de participación en la totalidad de los hechos es el nivel provincial, ya que realizó un $63 \%$ de las acciones protagonizadas por el orden gubernamental. Dentro del OGI provincial, los actores con mayor protagonismo fueron organismos pertenecientes al Poder Ejecutivo y directamente vinculados a la gestión de SAS, es decir el MASPyMA y el ENRESS, alcanzando un 56\% de la totalidad de acciones realizadas por el OGI provincial.

Cuadro 4.

Grado de participación de los distintos niveles del OGI en el proceso de producción social de SAS en la provincia de Santa Fe

\begin{tabular}{lc}
\hline \multicolumn{1}{c}{ OGl } & $\begin{array}{c}\text { Grado de partic ipación } \\
\text { en porcentaje }\end{array}$ \\
\hline Nivel provincial & $63 \%(47)$ \\
Nivel local & $29 \%(21)$ \\
Nivel nacional & $8 \%(6)$ \\
\hline Total & $100 \%$ \\
\hline
\end{tabular}

Fuente: elaboración propia.

El segundo agrupamiento en grado de importancia dentro del OGI, es el orden gubernamental institucional a nivel local (municipal o comunal), con un 29\% de las acciones realizadas por actores gubernamentales. La mayor cantidad de acciones de este grupo fueron realizadas por las autoridades del Poder Ejecutivo, comprendidas por el intendente municipal, el presidente comunal y funcionarios políticos.

Con un menor grado de participación, también estuvo presente en las acciones registradas el orden gubernamental a nivel nacional (8\%). Dentro de este agrupamiento, cabe resaltar el protagonismo del Ente Nacional de Obras Hídricas de Saneamiento (ENOHSA), que durante el período bajo estudio implementó diferentes programas para alcanzar las metas de cobertura en agua y saneamiento fijadas por los Objetivos de Desarrollo del Milenio, acordados por los países miembros de Naciones Unidas en el año 2000 (Orta, Portapila, Muñoz y Pérez, 2018).

Con relación a la participación de la sociedad civil, tuvo un protagonismo de un 33\% en la totalidad de los hechos registrados. El $87 \%$ de las acciones de este agrupamiento fueron realizadas por Identidades Político-Sociales. Dentro de este grupo, se destaca el protagonismo de las cooperativas de servicios públicos y la entidad federativa que las agrupa, con el $74 \%$ del total de acciones realizadas por la sociedad civil. 
Por último, nos encontramos con muy poca participación de actores individuales, $13 \%$ del total de los hechos realizados por la sociedad civil. Dentro de este sector, el poblamiento fue el que tuvo un poco más de protagonismo con un $8 \%$ de las acciones, considerando a vecinos con y sin acceso a servicios de agua y saneamiento.

3. ¿Cómo se intentaron resolver las distintas problemáticas relacionadas con la provisión de agua para consumo humano en Santa Fe?

Retomando nuestro interés por analizar cómo fueron las relaciones sociales entre los actores involucrados en el proceso de producción social de SAS, identificamos cuáles fueron las acciones que se pusieron en correspondencia. Para realizar un primer acercamiento al tipo de acciones decidimos distinguirlas en acciones con y sin confrontación para conocer el grado de conflictividad presente en el proceso. El 59\% de las acciones fueron sin confrontación y el $41 \%$ restante fueron hechos confrontativos (ver Cuadro 5).

Cuadro 5.

Hechos con y sin confrontación por tipo de acción y orden del problema

\begin{tabular}{|clccc|}
\hline & \multicolumn{4}{c}{ Orden (en porcentaje) } \\
\cline { 2 - 5 } & Tipo de acción & Socio-económico & Político & Total \\
\hline \multirow{2}{*}{$\begin{array}{c}\text { Sin confrontación } \\
\text { (68) } 59 \%\end{array}$} & Institucionalizada & 56 & 10 & 66 \\
& Enunciativa & 30 & 4 & 34 \\
\cline { 2 - 5 } & Total & 86 & 14 & 100 \\
\hline \multirow{3}{*}{$\begin{array}{c}\text { Con confrontación } \\
\text { (47) } 41 \%\end{array}$} & Institucionalizada & 19 & 15 & 34 \\
& Enunciativa & 45 & 19 & 64 \\
\cline { 2 - 6 } & Directa & 2 & 0 & 2 \\
\hline
\end{tabular}

Fuente: elaboración propia

Con relación al orden del problema, antes de analizar en qué porcentaje cada uno de ellos estuvo presente, nos interesa mostrar un detalle de los mismos. Entre los problemas de orden socio-económico cabe mencionar:

a) valor de la tarifa (aumento de la tarifa, obtención de subsidios, tipos de regímenes tarifarios);

b) medios de producción (reparación de infraestructura, realización de obras, ampliación de redes de acceso a los SAS, obtención de financiamiento, diferencias entre la macro y la micro medición);

c) balance económico del prestador de SAS (insuficiencia de los ingresos que se perciben por tarifas para hacer frente a los costos de producción);

d) acceso a servicios básicos (escasez de agua por sequías, garantizar la calidad y cantidad de agua, pérdidas en las redes de agua, demandas para que se garantice el acceso a servicios básicos);

e) salud (riesgo de contraer enfermedades por consumir agua de mala calidad);

f) contaminación del recurso hídrico (volcamiento de desechos cloacales, agrarios e industriales en cursos de agua superficiales) y;

g) demandas sociales (protestas de vecinos, reclamos de organizaciones, solicitudes de colegios profesionales). 
Por otro lado, con relación a los problemas de orden político, los clasificamos en:

a) modos de regulación del Estado (regulación y control de los prestadores de SAS, regímenes impositivos, modificación del marco regulatorio de SAS) y;

b) modos de intervención del Estado (falta de políticas públicas que solucionen el problema de acceso a SAS, críticas al modo en cómo acciona la fuerza política que ocupa el poder ejecutivo del Estado, dar a conocer a la ciudadanía los proyectos que pondrá en marcha el gobierno).

Ahora bien, nos interesa conocer cómo es la interrelación entre estas variables. En el Cuadro 5 se puede observar que de las acciones sin confrontación, 66\% de ellas fueron institucionalizadas y un $34 \%$ enunciativas. Con relación al problema que las origina podemos ver que en su mayoría se inscriben en el orden socioeconómico, con un $86 \%$.

Respecto al ámbito de las acciones de conflicto, podemos observar que predomina el acto enunciativo, con un $64 \%$ del total, lo cual estaría indicando que la confrontación se manifestó predominantemente en el campo discursivo, es decir que predominan más las críticas, quejas y reclamos a través de medios orales o escritos, que las acciones concretas mediante mecanismos institucionalizados o por hechos directos (movilizaciones, manifestaciones, decisión de dejar de pagar tarifas).

Si nos fijamos en el principal orden del problema que origina estas acciones, encontramos de nuevo una fuerte presencia de cuestiones socio-económicas (66\%) pero cobra mayor relevancia en la confrontación los problemas de orden político (34\%).

4. ¿Cómo actuó el gobierno provincial? ¿Cuál fue su estrategia selectiva?

Como lo propusimos en nuestros objetivos, nos interesa estudiar si hubo un cambio en el modo en cómo se relacionó la fuerza política que ocupó el ejercicio del PEP con relación a las localidades que son provistas por cooperativas. Es por eso que resulta significativo profundizar en el análisis del tipo de acciones que realizó y cuál fue el orden del problema que las originó.

En el Cuadro 6 podemos observar que un $68 \%$ de las acciones realizadas por el PEP fueron de carácter no confrontativo y el $32 \%$ restante fueron hechos de conflicto.

Cuadro 6.

Hechos con y sin confrontación realizados por el PEP

\begin{tabular}{cllccc}
\hline & & \multicolumn{3}{c|}{ Orden (en porcentaje) } \\
\cline { 2 - 5 } & Tipo de acción & Socio-económico & Político & Total \\
\hline \multirow{2}{*}{ Sin confuntacioín } & Institucionalizada & 57 & 11 & 68 \\
(28) 6fx & Enunciativa & 22 & 10 & 32 \\
\cline { 2 - 5 } & Total & 79 & 21 & 100 \\
\hline \multirow{2}{*}{ Con confuntación } & Institucionalizada & 23 & 31 & 54 \\
(13) 32X & Enunciativa & 15 & 31 & 46 \\
\cline { 2 - 5 } & Total & 38 & 62 & 100 \\
\hline
\end{tabular}

Fuente: elaboración propia. 
Dentro de las acciones sin confrontación, un $68 \%$ son institucionalizadas y el 32\% restante son enunciativas. Con relación al problema que origina estas acciones, un $79 \%$ son de orden socio-económico y un $21 \%$ de orden político. Teniendo un peso preponderante los problemas de orden socio-económico cuando el tipo de acciones que se emprenden son institucionalizadas, con un $57 \%$.

Si consideramos las acciones con confrontación podemos ver que el principal problema que las origina es de orden político (62\%), y que el tipo de acciones realizadas se distribuye casi por mitades entre acciones institucionalizadas (54\%) y enunciativas (46\%) (ver Cuadro 6). Dentro de las acciones originadas por problemas de orden político predominaron las que estuvieron dirigidas a regular y controlar el accionar de los prestadores de SAS, sobre todo lo relacionado con la adecuación a las normativas de calidad de agua.

Si consideramos sólo las acciones sin confrontación en su asociación con el tipo de problema que origina estas acciones, advertimos que predominan los problemas vinculados con obras de infraestructura (61\% en el Cuadro 7$)$. Este $61 \%$ de acciones se divide en institucionalizadas (43\%) y enunciativas (18\%). Las acciones enunciativas están relacionadas con declaraciones en actos públicos para anunciar la realización de obras o para inaugurar las ya realizadas. Las acciones institucionalizadas fueron de inversión en infraestructura y firmas de convenios para construcción de obra pública. Es decir que la construcción de infraestructura fue un modo predominante en el accionar del gobierno provincial para hacer frente a la problemática de acceso a SAS, pero las intervenciones realizadas no siguieron un plan sistemático y estructural. La estrategia principal consistió en la construcción de obras menores para mejorar la calidad del agua. Estas intervenciones tuvieron un carácter discrecional y discontinuo, no existiendo un plan sistemático que logre modificar el sistema discriminatorio de gestión de los SAS, más allá de los reclamos y exigencias de las autoridades locales, las cooperativas de SAS, los usuarios y los vecinos que no contaban con el servicio. ${ }^{[5]}$

Cuadro 7.

Hechos sin confrontación realizados por el PEP originados por problemas de obras de infraestructura

Problema que origina la acción en porcentaje

\begin{tabular}{lccc}
\cline { 2 - 4 } \multicolumn{1}{c}{ Tipo de acción } & $\begin{array}{l}\text { Obras de infraestruc tura } \\
\text { (medios de producción) }\end{array}$ & Otros & Total \\
\hline Instituc ionalizada 68\% (19) & 43 & 25 & 68 \\
Enunc iativa 32\% (9) & 18 & 14 & 32 \\
\hline Total & 61 & 39 & 100 \\
\hline
\end{tabular}

Fuente: elaboración propia.

En el Cuadro 8 podemos observar contra quiénes confrontó el gobierno provincial. En un $46 \%$ lo hizo contra otros actores institucionalesgubernamentales. Dentro de estos hechos, la confrontación es mayoritariamente de orden político (31\%).

En segundo lugar, en un 30\% confrontó contra prestadores de SAS. Estas acciones se dirigen al mismo tiempo a más de un prestador, incluyendo cooperativas, estados locales y ASSA. El problema que originó estas acciones fue 
en todos los casos de orden político, relacionado con cuestiones de regulación y control del accionar de los prestadores.

En tercer lugar, confrontó contra actores de la sociedad civil en un $24 \%$ de sus acciones, todas ellas originadas por problemas de orden socio-económico.

Cuadro 8.

Contra quiénes confrontó el gobierno provincial, por medio de qué tipo de acciones y cuáles fueron los problemas que las originaron

\begin{tabular}{llccc} 
& & \multicolumn{3}{c}{ Orden (en porcentaje) } \\
\cline { 3 - 5 } Contra quién & Tipo de acción & Socio-ec onómic o & Polític o & Total \\
\hline OGl $46 \%(6)$ & Instituc ionalizada & 15 & 16 & 31 \\
& Enunc iativa & 0 & 15 & 15 \\
\hline Prestador de SAS $30 \%(4)$ & Instituc ionalizada & 0 & 15 & 15 \\
& Enunc iativa & 0 & 15 & 15 \\
\hline \multirow{2}{*}{ SC 24\% (3) } & Instituc ionalizada & 8 & 0 & 8 \\
& Enunc iativa & 16 & 0 & 16 \\
\hline & Total & 39 & 61 & 100 \\
\hline
\end{tabular}

Fuente: elaboración propia.

Ahora bien, nos interesa observar cómo se manifestó la confrontación del gobierno provincial cuando confronta contra otros actores pertenecientes al ámbito estatal (identificado como OGI 46\% en el Cuadro 8). Una observación más desagregada nos permitió distinguir que un $50 \%$ de estas acciones se dirigieron a la fuerza política del PJ, que encabezaba el Poder Ejecutivo en algunas localidades de la provincia y presidía el Poder Ejecutivo Nacional. El 50\% restante se trata de acciones de confrontación al interior de la fuerza política del FPCyS. En este último caso, se evidencia un conflicto al interior de la fuerza gobernante, a través de hechos de confrontación originados por el ENRESS contra el gobernador y el MASPyMA, por el proyecto presentado para modificar el marco regulatorio de SAS, que pretendía restringir las funciones que posee el organismo de control.

En noviembre de 2010, el gobierno provincial presentó el proyecto de un nuevo marco regulatorio para los SAS de toda la provincia. El proyecto pretendía reemplazar la Ley 11220, vigente desde 1994. Entre los aspectos del nuevo marco regulatorio propuesto vinculados a la construcción de una lógica de gestión progresista, cabe mencionar los siguientes: (1) establecer un régimen normativo que reconozca el DHAS; (2) establecer un sistema de subsidios directos para usuarios en condiciones de vulnerabilidad social; (3) crear de un fondo provincial de saneamiento (proyecto de ley para modificar el marco regulatorio de SAS presentado por el PEP versión 2013).

Sin embargo, el proyecto de ley tenía aspectos controversiales con relación a la nueva función que debía tener el ENRESS y este fue el problema principal que dio lugar a las relaciones de confrontación que identificamos en el Cuadro 8. El proyecto presentado por el oficialismo proponía quitar al ente las funciones de regulación y mantener solamente funciones de control. ${ }^{[6]}$

La bibliografía que se especializa en el tema de la regulación de los servicios públicos, argumenta que su principal razón de ser no está en el carácter público o privado de la empresa prestataria, sino en el hecho de que se trata de monopolios naturales tradicionales, en los que por la naturaleza de los servicios prestados 
no es posible que haya más de una empresa prestadora por lo cual no se admite ningún tipo de competencia. Esto significa que un usuario de agua o cloacas no puede recurrir a otra empresa en caso de abusos o malos servicios, está compelido a mantenerse en la misma ya que no hay competencia posible, al no ser viable la duplicación de redes (Muñoz y Tagliavini, 2019). Debido a esta cuestión es imprescindible la existencia de un ente que tenga funciones de regulación y control y una composición que le otorgue independencia de quién es el prestador del servicio. Las demandas del ente regulador fueron tomadas por los miembros del bloque del FpV/PJ, que tenían mayoría en las dos cámaras legislativas del Poder Legislativo Provincial ${ }^{[7]}$.

5. ¿Cuáles fueron los actores que realizaron acciones de confrontación contra el gobierno provincial?

Siguiendo con los objetivos propuestos en este trabajo, nos interesa analizar cuáles fueron las acciones de confrontación dirigidas a los actores de la fuerza política gobernante. Para poder responder a éste objetivo, estudiaremos cuáles fueron los actores que protagonizaron las acciones de confrontación y el orden de los problemas que las originaron.

En el Cuadro 9 se puede observar que el $50 \%$ de las acciones de confrontación contra el PEP fueron realizadas por el OGI y el $50 \%$ por la SC.

\section{Cuadro 9.}

Tipo de actores que realizaron acciones de confrontación contra el PEP

\begin{tabular}{llccc} 
& & \multicolumn{3}{c}{ Tipo de orden (en porcentaje) } \\
\cline { 3 - 5 } & & Socio-ec onómico & Polític o & Total \\
\hline OGI 50\% (14) & PEP & 0 & 34 & 34 \\
& FpV/PJ & 58 & 8 & 66 \\
& Total & 58 & 42 & 100 \\
\hline SC 50\% (14) & IPS & 58 & 25 & 83 \\
& Al & 17 & 0 & 17 \\
& Total & 75 & 25 & 100 \\
\hline
\end{tabular}

Fuente: elaboración propia.

Entre los conflictos originados por reclamos y demandas dirigidas al gobierno provincial nos interesa destacar los siguientes: declaraciones críticas del presidente de la FeNCAP para que las localidades del interior cuenten con el mismo subsidio de ASSA; acciones protagonizadas por el ENRESS en contra del proyecto para modificar el marco normativo de SAS presentado por el gobierno provincial; reclamos, manifestaciones y realización de asambleas por parte de vecinos afectados por consumir agua con arsénico; reclamos de vecinos para que se extiendan las redes de agua y cloacas; reclamos de intendentes, presidentes comunales y cooperativas para abastecer de agua a las poblaciones afectadas por sequía; reclamos por parte de los prestadores de SAS al gobierno provincial para solicitar subsidios y poder adecuarse a la normativa de calidad de agua vigente; reclamos por parte de las autoridades políticas de las localidades que serían beneficiarias del programa provincial de acueductos, para que se acelere la construcción de los mismos y así poder solucionar de forma definitiva los problemas de calidad y cantidad de agua; declaraciones críticas por parte de legisladores de la oposición con relación al proyecto para modificar el marco 
regulatorio de SAS presentado por el oficialismo; elaboración por parte de miembros del bloque $\mathrm{PJ} / \mathrm{FpV}$ de un proyecto para modificar el marco regulatorio de SAS; entre otras.

Con relación al conflicto originado por el proyecto de marco regulatorio de SAS presentado por el oficialismo, los reclamos del ENRESS frente al intento del Gobierno de suprimir las funciones de regulación fueron tomados por los diputados provinciales opositores del FSFpT. Este bloque presentó en el Congreso provincial un proyecto de ley para modificar el marco regulatorio de SAS que mantenía las funciones de regulación y control del ENRESS (Proyecto de Ley, 2014, Expediente 29257 -DB-). En julio de 2014 el proyecto ingresó para su tratamiento a la Cámara de Diputados donde el FSFpT contaba con la mayoría. En setiembre de 2015 obtuvo media sanción y fue dirigido a la Cámara de Senadores. Sin embargo, el proyecto no fue tratado allí y perdió estado parlamentario $^{[8]}$.

Para finalizar este apartado, nos interesa señalar que las desigualdades en el acceso a los servicios de agua y saneamiento entre las localidades provistas por ASSA y las localidades provistas por cooperativas siguen estando presentes al cierre de nuestro período de estudio.

En el Cuadro 10 se puede observar que a pesar de las desigualdades presentes entre el subsistema ASSA y el subsistema cooperativas en cuanto a la asistencia que reciben por parte del Estado provincial, estas últimas han logrado avanzar en la cobertura de agua de un 80 a un $92 \%$ durante el período $2010-2018^{[9]}$. Sin embargo, siguen encontrándose 3 puntos porcentuales por debajo del nivel de cobertura de agua de las localidades provistas por ASSA.

\section{Cuadro 10.}

Avances en cobertura de agua y cloacas por tipo de prestador 2010-2018 (en porcentaje)

\begin{tabular}{|lcccc}
\hline & $\begin{array}{c}\text { Acceso a } \\
\text { agua por red } \\
2010\end{array}$ & $\begin{array}{c}\text { Acceso a } \\
\text { cloaca por } \\
\text { red 2010 }\end{array}$ & $\begin{array}{c}\text { Acceso a } \\
\text { agua por red } \\
2018\end{array}$ & $\begin{array}{c}\text { Acceso a } \\
\text { cloaca por } \\
\text { red 2018 }\end{array}$ \\
\hline ASSA & 90 & 50 & 95 & 68 \\
Cooperativas & 80 & 22 & 92 & 37 \\
\hline
\end{tabular}

Fuente: elaboración propia en base a CNPHV (2010) y ENRESS (2019).

Con relación a la cobertura de cloacas la desigualdad es aún más acentuada. Si bien las cooperativas han logrado ampliar la cobertura, pasando de un 22 a un $37 \%$, siguen estando muy por debajo del nivel de cobertura de las localidades provistas por ASSA, que era para el año 2018 de un $68 \%$.

\section{Conclusiones}

En el presente trabajo nos propusimos analizar si, a partir de que el FPCyS asumió el PEP en diciembre de 2007, comenzó a configurarse un modo distinto de relación entre el gobierno y las cooperativas. Para ver si se lograron superar o se siguen reproduciendo las desigualdades que describimos a lo largo del trabajo.

Para analizar el accionar del Estado nos propusimos realizar un abordaje desde el EER propuesto por Jessop, que conceptualiza al Estado como una relación social compleja. Constatamos la complejidad que posee el proceso 
social de producción de SAS en Santa Fe, debido a la diversidad de actores que están involucrados en las acciones que identificamos, tanto dentro del orden gubernamental institucional como de la sociedad civil.

Con relación a los conflictos que se suscitaron entre los actores que participaron y que estuvieron dirigidos a actores del PEP, hemos mencionado la diversidad de acciones institucionales y enunciativas que se realizaron para intentar influir sobre el curso de acción predominante de la fuerza política gobernante.

Respecto a la identificación de la estrategia selectiva impulsada por la fuerza política que ocupó el ejercicio del PEP, considerando las condiciones que contribuyeron a generar los otros actores sociales y políticos involucrados en el proceso (las acciones confrontativas que señalamos anteriormente), pudimos identificar que la estrategia principal consistió en la construcción de obras menores de infraestructura para mejorar la calidad del agua. Estas intervenciones tuvieron un carácter discrecional y discontinuo, no existiendo un plan sistemático que logre modificar el sistema discriminatorio de gestión de los SAS, más allá de los reclamos y exigencias de los prestadores locales, las cooperativas de SAS, los usuarios y los vecinos que no contaban con el servicio.

Asimismo, esta persistencia de una postura rígida por parte de los actores del PEP de no escuchar y no incorporar los reclamos de los actores sociales y políticos involucrados en el proceso social de producción de SAS, dificultó la posibilidad de lograr los consensos necesarios para modificar el marco regulatorio fijado por la Ley 11220. Como señalamos, el proyecto propuesto por el oficialismo presentaba aspectos progresivos y la elaboración de este proyecto respondía a algunas de las demandas realizadas por los actores políticos y sociales. Sin embargo, contenía aspectos regresivos en cuanto a las funciones que le corresponderían al ENRESS. Éste perdería sus funciones de regulación y mantendría solamente las de control. Lo que se pretendía en la práctica con este proyecto es que el ente no tuviera incidencia en el régimen tarifario y en la aprobación de los pedidos de aumento de tarifa de ASSA. La falta de consenso con los miembros del directorio del ENRESS y con la principal fuerza política de la oposición, obstaculizaron la posibilidad de avanzar en la modificación del marco regulatorio de SAS vigente, que reproduce una lógica discriminatoria de gestión de los SAS.

Con relación a las desigualdades en el acceso a SAS, se puede observar que a pesar de las dificultades que enfrentan las cooperativas, han logrado avanzar en la cobertura de agua de un $80 \%$ a un $92 \%$ durante el período 2010-2018. Sin embargo, siguen encontrándose 3 puntos porcentuales por debajo del nivel de cobertura de agua de las localidades provistas por ASSA. Con relación a la cobertura de cloacas la desigualdad es aún más acentuada. Si bien las cooperativas han logrado ampliar la cobertura, pasando de un 22 a un 37\%, siguen estando muy por debajo del nivel de cobertura de las localidades provistas por ASSA, que era para el año 2018 de un 68\%. Es decir que las desigualdades en el acceso a SAS en el territorio provincial siguen persistiendo. 


\section{Referencias}

ANTÓN, GUSTAVO (2010): Conflicto y poder en la Argentina: 2005-2008. Análisis de la emergencia del kirchnerismo como fuerza politica a partir de una lectura del diario La Nación (Tesis doctoral). Universidad de Buenos Aires, Buenos Aires.

BORRASTERO, CARINA (2018): «Elementos teóricos para la estructuración de un modelo de análisis socioeconómico del crecimiento de sectores industriales de perfil innovador», en: Intersticios sociales, 15, pp. 9-48.

CASTRO, ESTEBAN (2007): «El estudio interdisciplinario de los conflictos por el agua en el medio urbano: una contribución desde la sociología», en: Cuadernos del CENDES, 66, 24, pp. 21-46.

ENTE REGULADOR DE SERVICIOS SANITARIOS (2016): Listado de pequeños prestadores de servicios sanitarios (no concesionados por la provincia), Santa Fe, ENRESS.

ENTE REGULADOR DE SERVICIOS SANITARIOS (2019): Porcentajes de cobertura de agua y cloacas al 31/dic/2018, Santa Fe, ENRESS.

GOBIERNO DE LA PROVINCIA DE SANTA FE (2012): Plan Estratégico Provincial Santa Fe. Visión 2030, Santa Fe, Ministerio de Gobierno y Reforma del Estado de la Provincia de Santa Fe.

INSTITUTO NACIONAL DE ESTADÍSTICA Y CENSOS (2010): Censo Nacional de Población, Hogares y Viviendas 2010. Censo del Bicentenario. Resultados definitivos, Serie B, 2, Buenos Aires, INDEC.

INSTITUTO PROVINCIAL DE ESTADÍSTICAS Y CENSOS (2013): Producto Bruto Geográfico - 1993-2012. Resultados preliminares, Santa Fe, Ministerio de Economía, Secretaría de Planificación y Política Económica, Gobierno de Santa Fe.

JESSOP, BOB (2008a): «Poulantzas on the State as a Social Relation», en: Jessop, Bob. State Power: A Strategic-Relational Approach, Cambridge, Polity Press, pp. 118-139.

JESSOP, BOB (2008b): «The development of the Strategic-Relational Approach», en Jessop, Bob. State Power: A Strategic-Relational Approach, Cambridge, Polity Press, pp. 21-53.

JESSOP, BOB (2017): El Estado. Pasado, presente, futuro, Madrid, Catarata.

KLOSTER, KARINA (2016): Las luchaspor el agua en México 1990-2010. México DF, Universidad Autónoma de la Ciudad de México.

MARÍN, CARLOS (1984): Los hechos armados: Argentina 1973-1976, Buenos Aires, La Rosa Blindada.

MUÑOZ, ALBERTO; TAGLIAVINI, DAMIANO (2019): «Reestatización y regulación. Continuidades y rupturas del nuevo modelo de gobernabilidad de los servicios de agua y saneamiento en la Provincia de Santa Fe, Argentina», en: Castro, Esteban (comp.). Territorialidades del agua: conocimiento y acción para construir el futuro que queremos, Buenos Aires, Fundación CICCUS y WaterlatGobacit, pp. 182-207.

ORSOLINI, HUGO (2013): «El agua para consumo humano en la provincia de Santa Fe», en: XXIV Congreso Nacional del Agua, San Juan, 12 de septiembre.

ORTA, MELISA; PORTAPILA, MARGARITA; ANTÓN, GUSTAVO; FORTE, GUSTAVO (2020): «La calidad del agua para consumo humano como problemática social y política en Santa Fe, Argentina, 2007-2015. Una aproximación empírica», en: Hernández, Oliver y Alvarado, Armando (Eds.) 
¿Qué ley de aguas necesita México, para garantizar el derecho humano al agua?, México: Universidad Autónoma del Estado de México, pp. 113-140.

ORTA, MELISA; PORTAPILA, MARGARITA; MUÑOZ, ALBERTO; PÉREZ, IVÁN (2018): «El rol vital de las cooperativas como proveedoras de servicios de agua y saneamiento en la Provincia de Santa Fe, Argentina», en: WATERLATGOBACIT Working Papers, 5, pp. 33-74.

SECRETARÍA DE AGUAS (2009): Política hídrica santafesina. Marco de referencia. Santa Fe, Secretaría de Aguas, Ministerio de Aguas, Servicios Públicos y Medio Ambiente.

URCOLA, MARCOS; NOGUEIRA, ELENA; PORSTMANN, CARLOS; LATTUADA, MARIO (2015): «Desarrollo regional y territorios desiguales: elementos para un análisis de la provincia de Santa Fe», en: $I X$ Jornadas interdisciplinarias de estudios agrarios y agroindustriales argentinos $y$ latinoamericanos, Buenos Aires, 3-6 de noviembre.

\section{Notas}

[1] Para una descripción y análisis más detallado del proceso social que configuró el sistema actual de gestión de los servicios sanitarios en la provincia de Santa Fe, se puede consultar el trabajo realizado por Orta, Portapila, Muñoz y Pérez (2018).

[2] Entrevista realizada a la Directora de la Dirección General de Programas Estratégicos, Secretaría de Aguas, 01/07/2019.

[3] Como ejemplo, podemos mencionar los trabajos realizados por Marín (1984) y Antón (2010). Para el caso específico de la investigación de procesos de conflicto social vinculados con el recurso hídrico cabe mencionar el estudio de Kloster (2016).

[4] Se utilizaron las siguientes combinaciones de palabras clave: calidad agua arsénico santa fe; calidad agua santa fe; agua cooperativa; provisión agua; salud agua; agua agroquímicos; tarifa agua; agua reclamo y agua conflicto. El buscador utilizado fue Google y se realizaron búsquedas específicas en los sitios web del Diario El Litoral (site:ellitoral.com) y el Diario La Capital (site:lacapital.com.ar).

[5] Entrevista a Presidente del Ente Regulador de los Servicios Sanitarios, 11/07/17; entrevista al Presidente de la Cooperativa de Obras, Servicios Públicos, Asistenciales y Vivienda de Carcarañá, 17/10/2016; entrevista a Presidente de Unión de Usuarios y Consumidores, 23/01/2018.

[6] Entrevista realizada a secretario de Aguas, Ministerio de Aguas, Servicios Públicos y Medio Ambiente, 18/10/2016; entrevista a Presidente de ASSA, 16/10/2017.

[7] A partir de las elecciones de 2011, se da una situación particular en el poder legislativo relacionada con que el oficialismo no obtiene la mayoría en ninguna de las dos Cámaras. La mayoría en ambas cámaras fue obtenida por el Frente Santa Fe para Todos (FSFpT), integrado por el PJ y el FpV.

[8] Entrevista realizada a diputado de la provincia de Santa Fe por el FpV, 25/10/2019; entrevista realizada a director ejecutivo del Centro de Educación, Servicios y Asesoramiento al Consumidor (CESyAC), 11/07/2017.

[9] Para finalizar el período de estudio pusimos datos del año 2018, no del 2015, puesto que los datos provistos por el ENRESS son para el año 2018. El organismo no cuenta con un registro sistemático de datos de cobertura de SAS anterior a esta fecha. 